\title{
Worst-Case Optimal Scheduling and Real-Time Control of a Microgrid Offering Active Power Reserve
}

\author{
${ }^{1}$ University of Zagreb, Faculty of Electrical Engineering and Computing, Department of Control and Computer Engineering, Laboratory \\ for Renewable Energy Systems, Unska 3, 10000 Zagreb, Croatia
}

\begin{abstract}
This work is focused on optimization problems within the predictive control framework for determining and engaging the flexibility of a microgrid in grid-microgrid energy exchange. The microgrid has a controllable battery storage and other components represented with a residual power flow. All major economic constituents of the grid-connected microgrid operation are considered: day-ahead, intra-day, peak power and battery degradation costs, as well as rewards and penalties for providing flexibility. The problems are posed as linear worst-case minimization programs in which all flexibility activation scenarios are taken into account. An analysis is conducted for various combinations of flexibility reservation and activation prices that can be bid to a grid entity. The technical and economic feasibility of the flexibility provision is confirmed by the use of an online model predictive controller that optimally meets the requirements of grid flexibility according to the declared reserve in the environment of online occurring disturbances and events.
\end{abstract}

Keywords: smart grids, microgrids, linear programming, model predictive control, demand response

\section{Introduction}

Frequency regulation as one of the means to ensure optimal functioning of the electrical grid can be divided into three categories based on the response time of the regulation units: primary, secondary and tertiary. Tertiary regulation can be further divided into tertiary regulation for system balancing and tertiary regulation for system safety. This paper focuses on the tertiary regulation of frequency for system balancing achieved through the activation of active power reserves by a transmission system operator (TSO). The TSO accepts or declines the declared power reserve under the offered pricing conditions. In case of acceptance, the entity must change its previously planned and declared electrical load upon the TSO request within a time frame that differs from contract to contract [1]. Usual time frames discussed in this area of research include requests coming as immediate as 15 -minute prior to activation according to arrangements for a specific time slot made the day before. In this work we focus on the power reserve activation that can be called by the TSO up to 15 minutes before the activation time according to the declaration made beforehand. This behavior is also termed as explicit demand response - a framework for a TSO or a distribution system operator (DSO) to offer contract deals to electrical energy consumers with the aim of costs reduction on both sides based on flexibility of the end-consumers' consumption [2]. 
Besides explicit demand response, the electrical energy provider can implicitly influence consumers to reduce peak power during times that are characterized by high electrical energy usage. This is achieved by increasing prices of electrical energy in peak times and decreasing prices when it is strategically appropriate to encourage consumption. Additionally, every electrical energy consumer contractually agrees with the electrical energy provider on a specific maximum power. The consumption above the declared maximum power is penalized by higher electrical energy prices [3].

To allow consumers or prosumers participation in flexibility provision, novel control systems enabling efficient and profitable demand response services have been developed. In [4] frequency regulation system for DR using electric vehicles charging and historical data to determine expectations of stochastic variables is developed. Cost-benefit analyses, using mixed integer linear programming, for several microgrid configurations are given in [5]. Besides a battery storage system, a building thermal mass can also be used as a thermal energy storage that contributes to the building's flexibility in electrical energy consumption planning. The downside of the approach are larger thermal losses compared to the optimal control focused solely on energy-efficiency [6]. The coordinated operation between the building microgrid and the central heating, ventilation and air conditioning system (HVAC) for mutual flexibility provision is explored in our previous work [7].

This paper is a shortened version of the work [8]. Its main contributions are summarized as:

- optimization-based determination of optimal frequency regulation reserve power offer according to commercial rules for flexibility provision by the Croatian TSO,

- real-time Model Predictive Controller (MPC) that assures feasibility of flexibility provision for every possible moment of activation,

- worst-case optimization without stochastic data needed.

The work is organized as follows: Section 2 introduces a considered building microgrid and explains the DR scheme together with its corresponding optimization problem formulation. The simulation results based on a real case-study are elaborated in Section 3 and the conclusion is given in the final section.

\section{Formulation of the optimization problem}

\section{A. Microgrid description}

The considered microgrid consists of a battery storage and a non-controllable consumption combined with a photovoltaic source. Since the microgrid is connected to a distribution grid, its energy exchange with the grid is described with:

$$
E_{\mathrm{g}}(k)=u_{\mathrm{ch}}(k)-u_{\mathrm{dch}}(k)+E_{\mathrm{nc}}(k),
$$

where positive values of $E_{\mathrm{g}}(k)$ relate to the energy taken from the grid and negative values to the energy provided to the grid, $u_{\mathrm{ch}}$ and $u_{\mathrm{dch}}$ are controllable battery charging and discharging energies, respectively. Notation $E_{\mathrm{nc}}$ stands for the non-controllable energy consumption of the building. All energy variables in discrete-time actually correspond to the energies in time intervals between $k T$ and $(k+1) T$ where $T$ is the discretization time of $15 \mathrm{~min}$. System dynamics is described with only one system state which is the battery state of energy:

$\operatorname{SoE}(k+1)=\operatorname{SoE}(k)+\eta u_{\mathrm{ch}}(k)-u_{\mathrm{dch}}(k) / \eta$

where $\eta$ is the efficiency of the battery system (power converter + battery). As in [10], it is possible for the battery to be both charged and discharged within one discretization interval, but respecting the following constraints:

$$
\left\{\begin{array}{c}
u_{\mathrm{ch}}(k)+u_{\mathrm{dch}}(k) \leq P_{\max } T \\
0 \leq u_{\mathrm{ch}}(k) \leq P_{\max } T \\
0 \leq u_{\mathrm{dch}}(k) \leq P_{\max } T
\end{array}\right.
$$

where $P_{\max }$ denotes the maximum power of the battery power converter $(9.6 \mathrm{~kW}$ in the considered microgrid).

\section{B. Cost variables}

In this subsection components of the microgrid cost function for energy exchange with the grid including DR functionality are introduced. These components include day-ahead energy cost, intra-day energy cost, peak power penalization, frequency regulation reserve power revenue, regulation energy revenue and battery degradation cost.

Consumed electrical energy cost $J_{\mathrm{da}}$ is calculated in the following way:

$$
J_{\mathrm{da}}\left(E_{\mathrm{g}}\right)=\sum_{k} c_{\mathrm{da}}(k) E_{\mathrm{g}}(k)
$$

where $c_{\mathrm{da}}$ is a vector of day-ahead prices for every 15-min discretization interval, obtained from the supplier or the electricity market.

On the intra-day market, the deviation of the exhibited energy exchange profile $E_{\mathrm{g}}$ from the day-ahead predicted/ declared reference energy profile $E_{\text {g,ref }}$ is penalized with the cost function:

$$
J_{\text {id }}\left(E_{\mathrm{g}}, E_{\mathrm{g}, \text { ref }}\right)=\sum_{k} 1.2 c_{\mathrm{da}}(k)\left|E_{\mathrm{g}}(k)-E_{\mathrm{g}, \mathrm{ref}}(k)\right|
$$

Depending on the optimization problem, $E_{\mathrm{g}, \mathrm{ref}}$ is either a profile to be declared to the grid that is an optimization 
variable or an already declared profile which is then a constant parameter.

The microgrid contracts peak power $P_{\mathrm{pp}, \mathrm{c}}$ to the grid on monthly basis. The peak power cost considered in this paper is derived based on the peak power billing in Croatia [9], [10] and is defined with

$$
\text { s.t. }\left\{\begin{array}{c}
J_{\mathrm{pp}}\left(E_{\mathrm{g}}\right)=c_{\mathrm{pp}} \varepsilon_{\mathrm{pp}} \\
\varepsilon_{\mathrm{pp}} \geq \varepsilon_{\mathrm{pp}, \mathrm{past}} \\
\varepsilon_{\mathrm{pp}} \geq 0.85 P_{\mathrm{pp}, \mathrm{c}} \\
\varepsilon_{\mathrm{pp}} \geq E_{\mathrm{g}}(\mathrm{k}) / T \\
\varepsilon_{\mathrm{pp}} \geq 3 E_{\mathrm{g}}(\mathrm{k}) / T-2.1 P_{\mathrm{pp}, \mathrm{c}}
\end{array}\right.
$$

where $\mathcal{E}_{p p}$ is an auxiliary variable and $c_{p p}$ is the price of peak power obtained from the grid operator.

The microgrid contracts unique reserve power $P_{\text {res }}$ for every day in the next week and it is rewarded with

$J_{\text {res }}\left(P_{\text {res }}\right)=\sum_{d} c_{\text {res }} \operatorname{sgn}\left(P_{\text {res }}(d)\right) P_{\text {res }}(d), \forall d \in \mathcal{W}(7)$

where $c_{\text {res }} \leq 0$ is the price of reserve active power and $\mathcal{W}$ denotes the set of indices of days in a week. Since reserve power market is performed as an auction, choosing $c_{\text {res }}$ is out of the scope of this paper and the reader can find more about this problem in e.g. [11].

If the grid activates a part of or the whole agreed flexibility reserve, which can last up to two hours, the microgrid is rewarded for the exhibited difference in electrical energy consumption compared to the declared consumption:

$$
\begin{array}{r}
J_{\mathrm{act}}\left(E_{\mathrm{g}}, E_{\mathrm{g}, \mathrm{ref}}, P_{\mathrm{act}}, i\right)=\sum_{k} c_{\mathrm{act}} \varepsilon_{\mathrm{act}}(k), i \leq k<i+8 \\
\text { s.t. }\left\{\begin{array}{c}
\varepsilon_{\mathrm{act}}(k) \leq \operatorname{sgn}\left(P_{\mathrm{act}}\right)\left(E_{\mathrm{g}}(k)-E_{\mathrm{g}, \mathrm{ref}}(k)\right) \\
\varepsilon_{\mathrm{act}}(k) \leq \operatorname{sgn}\left(P_{\mathrm{act}}\right) P_{\mathrm{act}} \mathrm{T} \\
\varepsilon_{\mathrm{act}}(k) \geq \operatorname{sgn}\left(P_{\mathrm{act}}\right)(1-\alpha) P_{\mathrm{act}} \mathrm{T}
\end{array}\right.
\end{array}
$$

where $P_{\text {act }}$ is a regulation power request of the grid that must be of the same sign and in absolute value lower than the absolute value of $P_{\text {res, }}, \mathcal{E}_{\text {act }}$ is an auxiliary variable, $c_{\text {act }}$ is the price of regulation energy, $\alpha=0.25$ is a tolerance factor.

Battery capacity is degraded by every charging and discharging action which is penalized with:

$$
J_{\mathrm{bd}}\left(u_{\mathrm{ch}}, u_{\mathrm{dch}}\right)=\sum_{k} c_{\mathrm{bd}}\left(u_{\mathrm{ch}}(k)+u_{\mathrm{dch}}(k)\right)
$$

where $c_{\mathbf{b d}}$ is the battery degradation cost [12].

\section{Offline analysis optimization problem}

The considered optimization problem consists of one scenario $S_{i}$ for the activation at every possible discretization interval $i$ in a day $(i \in \mathcal{H}, \mathcal{H}=\{0,1, \ldots, 95\})$ and of a scenario $S_{\mathrm{n}}$ without activation. Further on, indices $i$ and $\mathrm{n}$ are used with different variables to denote a scenario to which a particular variable belongs. The information about the activation at the moment $i$ is available just at the interval $i-1$ which means that all states of the scenario $S_{i}$ must be equal to the ones of the scenario $S_{\mathrm{n}}$ when the activation occurs. Such an optimization problem can be qualified as the worst-case multi-stage recourse problem according to [13].

Constraints that connect scenarios $S_{\mathrm{n}}$ and $S_{i}$ assure that all decision variables are calculated using only information available at the corresponding moment:

$$
\begin{aligned}
& \operatorname{SoE}_{i}(k)=\operatorname{SoE}_{\mathrm{n}}(k) \forall k \\
& \quad \in\{i+96 d \mid d \in \mathcal{W}, i \in \mathcal{H}\}
\end{aligned}
$$

Scenario $S_{i}$ contains seven activations at the $i^{\text {th }}$ adiscretization interval each day (every $24 \mathrm{~h}$ ), which is athe most frequently possible and the scenario $S_{\mathrm{n}}$ does anot contain any activation. Altogether there are 97 ascenarios. Total costs $J_{\mathrm{n}}$ of scenario without activation aand $J_{i}$ of scenarios with activation at interval $i$ are adefined as:

$$
\begin{gathered}
J_{\mathrm{n}}=J_{\mathrm{da}}\left(E_{\mathrm{g}, \mathrm{n}}\right)+J_{\mathrm{pp}}\left(E_{\mathrm{g}, \mathrm{n}}\right)+J_{\mathrm{bd}}\left(u_{\mathrm{ch}, \mathrm{n}}, u_{\mathrm{dch}, \mathrm{n}}\right) \\
J_{i}=J_{\mathrm{da}}\left(E_{\mathrm{g}, i}\right)+J_{\mathrm{pp}}\left(E_{\mathrm{g}, i}\right)+J_{\mathrm{bd}}\left(u_{\mathrm{ch}, i}, u_{\mathrm{dch}, i}\right)+ \\
J_{\mathrm{id}}\left(E_{\mathrm{g}, i}, E_{\mathrm{g}, \mathrm{n}}\right)+\sum_{d} J_{\mathrm{act}}\left(E_{\mathrm{g}, i}, E_{\mathrm{g}, \mathrm{n}}, P_{\mathrm{res}, i}, i+96 d\right)
\end{gathered}
$$

It can be seen from (13) that every scenario assumes the grid will activate the whole contracted reserve power $P_{\text {res }}$. The optimization variables of the offline problem, besides the auxiliary variables from (6), (8) and (9), are $u_{\mathrm{ch}}, u_{\mathrm{dch}}, P_{\mathrm{pp}, \mathrm{c}}$ and $\operatorname{SoE}(0)$ of all scenarios and the vector of contracted daily regulation power reserve $P_{\text {res }}$ while the cost being minimized is:

$$
J_{\mathrm{offline}}^{*}=\min _{u_{\mathrm{ch}}, u_{\mathrm{dch}}, S O E(0), P_{p p, c}, P_{\mathrm{res}}} J_{\text {res }}+J_{\mathrm{worst}}
$$

$$
\text { s.t. }\left\{\begin{array}{c}
J_{\text {worst }} \geq J_{\mathrm{n}} \\
J_{\text {worst }} \geq J_{i}, \forall i \\
(1)-(13)
\end{array}\right.
$$

\section{Online MPC optimization problem}

Online MPC operates in receding horizon fashion with a sampling time $T=15 \mathrm{~min}$ and applies only the optimal control variables for the first time-instant $k, u_{\mathrm{ch,n}}(0)$ and $u_{\text {dch,n }}(0)$, to the battery storage system.

Contrary to the offline formulation, $J_{\text {act }}$ is defined as follows for the online formulation:

$$
\begin{gathered}
J_{\mathrm{act}}\left(E_{\mathrm{g}}, E_{\mathrm{g}, \mathrm{ref}}, P_{\mathrm{act}}, i\right)=\sum_{k} c_{\mathrm{act}} \varepsilon_{a c t}(k)+ \\
\sum_{k} c_{\mathrm{s}} \varepsilon_{\mathrm{s} 1}(k), \quad i \leq k<i+8,
\end{gathered}
$$




$$
\text { s.t. }\left\{\begin{array}{c}
\varepsilon_{\mathrm{act}}(k) \leq \operatorname{sgn}\left(P_{\mathrm{act}}\right)\left(E_{\mathrm{g}}(k)-\gamma(i, k) E_{\mathrm{g}, \mathrm{ref}}(k)\right), \\
\varepsilon_{\mathrm{act}}(k) \leq \operatorname{sgn}\left(P_{\mathrm{act}}\right) P_{\mathrm{act}} T, \\
\varepsilon_{\mathrm{act}}(k)+\varepsilon_{\mathrm{s} 1}(k) \geq \operatorname{sgn}\left(P_{\mathrm{act}}\right)(1-\alpha) P_{\mathrm{act}} T, \\
\gamma(i, k)=\left\{\begin{array}{c}
\sum_{j=-4}^{1} E_{\mathrm{g}}(i+j) \\
\sum_{j=-4}^{1} E_{\mathrm{g}, \mathrm{ref}}(i+j) \\
1, k>k_{\mathrm{mid}}
\end{array}, k \leq k_{\mathrm{mid}},\right.
\end{array}\right.
$$

The introduced auxiliary variable $\varepsilon_{\mathrm{s} 1}$ and its contribution to $J_{\text {act }}$ are an implementation of soft constraints to enable feasibility of the optimization problem even if the microgrid cannot fulfill activated regulation power due to an unfavorable noncontrollable consumption. Soft constraint penalty is taken as $c_{\mathrm{s}}=10^{5}$.

Both the declared consumption profile $E_{\mathrm{g}}^{*}$ and the profile that is going to be declared $E_{\mathrm{g}, \mathrm{n}}^{+}$are used as a reference profile in (16), depending on the discretization interval $k$. The prediction horizon in on-line MPC always corresponds to the length of known day-ahead prices. When the prices for the following day are announced, the online MPC abruptly increases the prediction horizon for another 24 hours and declares the solution $E_{\mathrm{g}, \mathrm{n}}^{+}$for the following day to the grid. Notation $k_{\text {mid }}$ denotes the last discretization interval with the known declared consumption profile. To avoid nonlinearity and the need for a sequential linear program it is assumed that after $k_{\text {mid, }} \gamma$ is equal to 1 as the worst case, and thus an auxiliary cost $J_{\text {aux }}$ and constraints are added to scenario $S_{\mathrm{n}}$ :

$$
\begin{gathered}
J_{\mathrm{aux}}\left(E_{\mathrm{g}, \mathrm{n}}, E_{\mathrm{g}, \mathrm{ref}}\right)=\sum_{k} c_{\mathrm{s}} \varepsilon_{\mathrm{s} 2}(k), \\
\text { s.t. }\left\{\begin{array}{c}
\operatorname{sgn}\left(P_{\mathrm{res}}\right)\left(E_{\mathrm{g}, \mathrm{n}}(k)+\varepsilon_{\mathrm{s} 2}(k)\right) \leq \operatorname{sgn}\left(P_{\mathrm{res}}\right) E_{\mathrm{g}, \mathrm{ref}}(k) \\
\varepsilon_{\mathrm{s} 2}(k) \geq 0, \\
\forall k \in\left\{k_{\text {mid }}-10, \ldots, k_{\text {mid }}\right\}
\end{array}\right.
\end{gathered}
$$

All cost functions are the same as in the offline problem except $J_{\text {act }}$ which is then added to (12) and (13), and also $J_{\text {aux }}$ and $J_{\text {id }}\left(E_{\mathrm{g}, \mathrm{n},}^{-}, E_{\mathrm{g}}^{*}\right)$ are added to (12).

The final problem to be solved is

$$
\begin{gathered}
J_{\text {online }}^{*}=\min _{u} J_{\text {worst }} \\
\text { s.t. }\left\{\begin{array}{c}
J_{\text {worst }} \geq J_{\mathrm{n}}, \\
J_{\text {worst }} \geq J_{i}, \forall i \\
(1)-(6),(10)-(13),(16)-(19)
\end{array}\right.
\end{gathered}
$$

where $u$ denotes the charging and discharging signals of all scenarios.

\section{Simulation}

\section{A. Offline analysis}

In the following section, offline analysis is conducted to ascertain how the agreed activation and reservation prices between the TSO and the microgrid affect operational costs of the microgrid, based on historical data $E_{\text {nc }}$ and $c_{\text {da. }}$.

In Table I, several combinations of activation prices and reservation prices are shown for the maximum activation duration of one and two hours, respectively. To make sure that the reserved powers are at their maximum and uniform across all seven days, it is recommended to raise the reservation price instead of the activation price to further increase the optimal reserved powers. Of the other results listed in the table, $\bar{J}$ denotes the mean cost of all scenarios, and $J_{\text {worst }}$ denotes the highest cost value among all the scenarios that generally corresponds to the scenario $S_{\mathrm{n}}$ since it does not contain the activation reward $J_{\text {act. }}$.

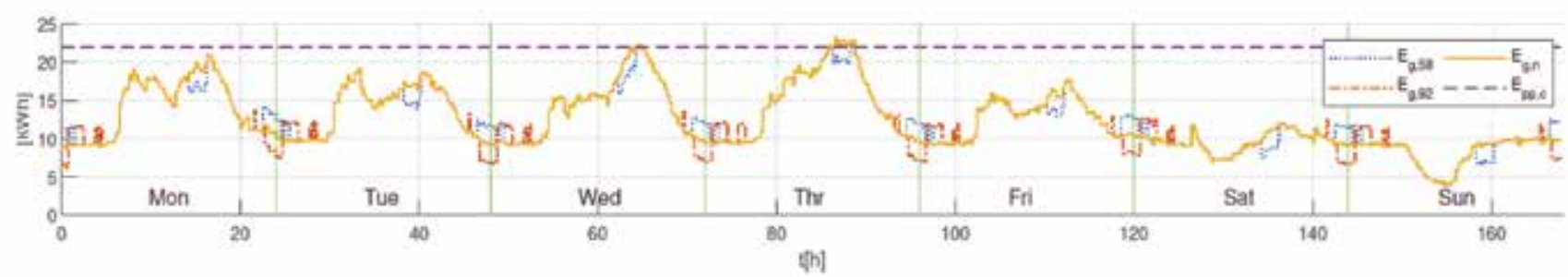

(a)

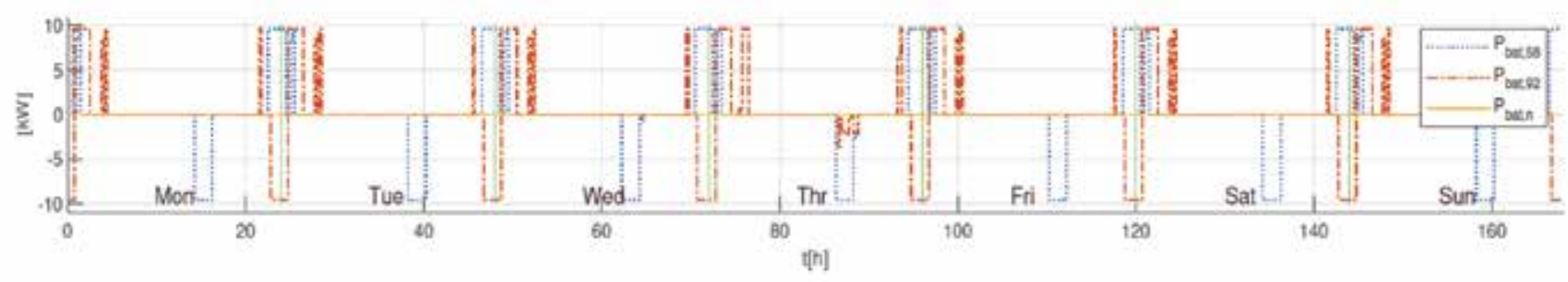

(b)

Fig. I: Results of offline analysis, (a) energy exchange with the grid and (b) battery charging power $\left(P_{\text {hat }}=\frac{u_{b}-u_{\text {abh }}}{T}\right)$. 
TABLE I: Offline analysis results.

\begin{tabular}{|c|c|c|c|}
\hline Prices & $P_{\text {res }}[\mathrm{kW}]$ & $\bar{J}[€]$ & $J_{\text {worst }}[€]$ \\
\hline $\begin{array}{c}\mathrm{c}_{\mathrm{act}}=-0.4 € / \mathrm{kWh} \\
\mathrm{c}_{\mathrm{res}}=-0.1 € / \mathrm{kW}, \mathrm{lh}\end{array}$ & $\begin{array}{c}{[-12.8,-12.8,-9.85,} \\
-5.23,-12.8,-12.8, \\
-12.8]\end{array}$ & 617.34 & 621.83 \\
\hline $\begin{array}{c}\mathrm{c}_{\mathrm{act}}=-0.4 € / \mathrm{kWh} \\
\mathrm{c}_{\mathrm{res}}=-0.7 € / \mathrm{kW}, 1 \mathrm{~h}\end{array}$ & $\begin{array}{c}{[-12.8,-12.8,-12.8,} \\
-12.8,-12.8,-12.8, \\
-12.8]\end{array}$ & 563.88 & 570.63 \\
\hline $\begin{array}{c}\mathrm{c}_{\mathrm{act}}=-0.4 € / \mathrm{kWh} \\
\mathrm{c}_{\mathrm{res}}=-0.1 € / \mathrm{kW}, 2 \mathrm{~h}\end{array}$ & $\begin{array}{c}{[-12.8,-12.8,-9.85} \\
-5.23,-12.8,-12.8, \\
-12.8]\end{array}$ & 613.17 & 621.47 \\
\hline $\begin{array}{c}\mathrm{c}_{\mathrm{act}}=-0.4 € / \mathrm{kWh} \\
\mathrm{c}_{\mathrm{res}}=-0.7 € / \mathrm{kW}, 2 \mathrm{~h}\end{array}$ & $\begin{array}{c}{[-12.8,-12.8,-12.8,} \\
-12.8,-12.8,-12.8, \\
-12.8]\end{array}$ & 559.64 & 570.25 \\
\hline without DR & & & 625.64 \\
\hline
\end{tabular}

Fig. 1a. shows energy exchange with the grid for a period of seven days with a time resolution of 15 -minutes. It can be noticed that the energy exchange profile $E_{\mathrm{g}, \mathrm{n}}$ does not include any charges or discharges of the battery because this strategy enables the microgrid to fulfill all the activations in scenarios $S_{i}$. In Fig. 1b, it can also be noticed that the batteries in the scenarios $S_{i}$ tend to charge when the electricity prices are low (usually around midnight) and discharge to their full extent (2 hours and $75 \%$ of the reserved power in the particular simulation) when they are activated. The activation that starts around midnight of the last day is circled back to the first day in the optimization problem to satisfy the repeatability condition of the battery's $S o E$.

Contracted peak energy $E_{\mathrm{pp}, \mathrm{c}}=P_{\mathrm{pp}, \mathrm{c}} T$ can also be found in the graph (purple dashed). It is chosen so that the peak power of scenario $S_{\mathrm{n}}$ is on the edge of the interval where there are no penalties, i.e. it does not cross $105 \%$ of $P_{\mathrm{pp}, \mathrm{c}} T$. The energy exchange profile $E_{\mathrm{g}, 92}$ of the scenario $S_{92}$ (flexibility activation at 22:45 every day) uses the battery to reduce the maximum total peak power while profile $E_{\mathrm{g}, 58}$ exhibits flexibility activation at the same time.

\section{B. Online MPC}

One pair of prices $c_{\text {res }}=-0.7 \quad E U R / k W$ and $c_{\text {act }}=-0.4 \mathrm{EUR} / \mathrm{kWh}$ and the calculated optimal $P_{\text {res }}=-12.8 \mathrm{~kW}$ are taken for a case study simulation of the online MPC.

The simulation results are shown in Figs. $2 a$ and $2 b$. In the online simulation optimization is run in every discretization interval and all signals are marked with a suffix denoting the interval when they are obtained. The number in the index denotes a scenario relative to the interval while $E_{\mathrm{g}, \mathrm{cl}}$ denotes the exhibited energy exchange profile obtained with the closed-loop MPC.

It can be seen how the microgrid changes its plan during the time. At the beginning of the simulation the microgrid decides to charge the battery around midnight in the case of scenario $i=52$ (dashed dark blue line $E_{\mathrm{g}, 52}^{0}$ and $P_{\text {bat,52). Midnight battery charging corresponds to the }}^{0}$ low energy prices period and it is within the recuperation period, assuring the microgrid will be ready for every possible next activation.

After the activation occurred at $k=52$ (13:00) the microgrid does not have to be ready for an activation until $k=52+96$ and reference profile $E_{\mathrm{g}, \mathrm{d}+1}^{*}$ is not declared until 17:00. The microgrid exploits that situation to postpone charging to expiration of the recuperation period which can be seen by observing $E_{\mathrm{g}, \mathrm{d}+1}^{*}$ (yellow dashed line). Thus the microgrid reduced the battery degradation cost for the first several scenarios after the recuperation period, which is considerably more than a difference in electrical energy prices. Not only frequency regulation is fully fulfilled without discharging the battery but also one charging cycle is avoided. Furthermore, even if the next activation is after the rescheduled charging (i.e. after $40 \mathrm{~h}$ ), the microgrid still achieved savings by avoiding intra-day costs that would occur in the case of midnight charging.
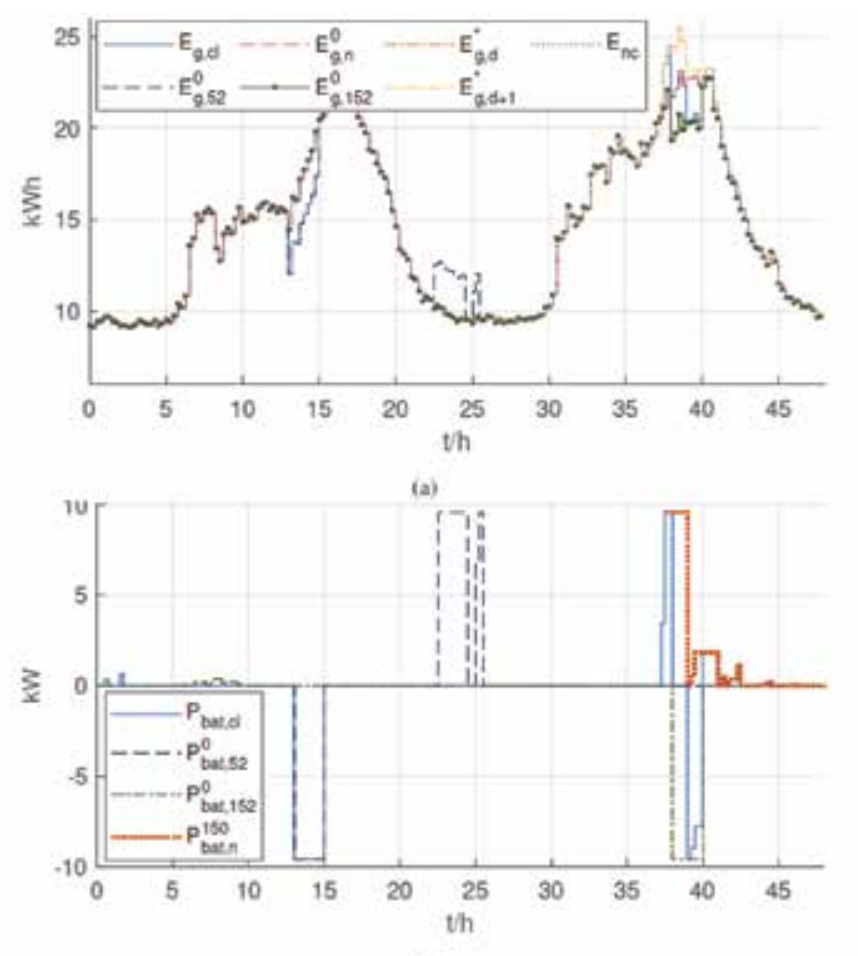

(b)

Fig. 2: Simulation results for real-time MPC, (a) energy exchange with the grid and (b) battery charging power $\left(P_{\mathrm{bat}}=\frac{\text { wox }_{\mathrm{a}}-w_{\mathrm{at}}}{\mathrm{T}}\right)$

\section{Conclusion}

This paper deals with the building microgrid's ability to participate in tertiary frequency regulation through demand response and obtaining benefits by the predictive control of battery charging and discharging. At the same time additional benefits are assured through peak power reduction and participation in the day-ahead energy market. Furthermore, the building contributes to better grid operation and power system regulation. 


\section{Acknowledgment}

This work was supported by European Union Regional Development Fund via Operational Programme Competitiveness and Cohesion for Croatia through the Project PC-ATE Buildings - Development of a System for Predictive Control and Autonomous Trading of Energy in Buildings under Contract KK.01.2.1.01.0069.

\section{References}

[1.] Croatian Transmission System Operator. (2020) Demand side response. [Online]. Available: https://www.hops.hr/en/ demand-side-response

[2] P. Zancanella, P. Bertoldi, and B. Kiss, "Demand response status in EU member states," Publications Office of the European Union, 2016.

[3] B. Brkić and M. Vašak, "Analysis of a diesel-generatorassisted peak power reduction in a production plant," in 2020 IEEE 11th International Symposium on Power Electronics for Distributed Generation Systems (PEDG), 2020, pp. 242246.

[4] E. Sortomme and M. A. El-Sharkawi, "Optimal scheduling of vehicle-to-grid energy and ancillary services," IEEE Transactions on Smart Grid, vol. 3, no. 1, pp. 351-359, 2012.

[5] F. Marangoni, L. Magatao, and L. de Arruda, "Demand response optimization model to energy and power expenses analysis and contract revision," Energies, vol. 13, no. 11, p. 2803, 2020

[6] L. Kreuder and C. Spataru, "Assessing demand response with heat pumps for efficient grid operation in smart grids," Sustainable Cities and Society, vol. 19,pp.136-143, 2015.
[Online]. Available: http://www.sciencedirect.com/science/ article/pii/S2210670715300159

[7] M. Kovačević, D. Marušić, and M. Vašak, "Price-optimal electrical and thermal energy flow control within microgrid - smart grid interaction", in 2020 IEEE 11th International Symposium on Power Electronics for Distributed Generation Systems (PEDG), 2020, pp. 247-252.

[8] M. Kovačević, B. Brkić, and M. Vašak, "Worst-case optimal scheduling and real-time control of a microgrid offering active power reserve," 2021 23rd International Conference on Process Control (PC), 2021, pp. 66-71, doi: 10.1109/ PC52310.2021.9447502.

[9] Croatian Energy Regulatory Agency. (2020) Distribution system tariff. [Online]. Available: https://zakon.poslovna. $\mathrm{hr} /$ public/tarifni-sustav-za-distribuciju-elektricneenergije\%2C-bez-visine-tarifnih-stavki/409408/zakoni.aspx

[10] HEP-Operator distribucijskog sustava d.o.o. (2020) Distribution system prices. [Online]. Available: https:// www.hep.hr/ods/kupci/poduzetnistvo/tarifne-stavkecijene-161/161\#

[11] M. Shafie-khah, M. Moghaddam, M. Sheikh-El-Eslami, and J. Catalao, "Optimised performance of a plug-in electric vehicle aggregator in energy and reserve markets," Energy Conversion and Management, vol. 97, pp. 393 - 408, 2015.

[12] F. Rukavina and M. Vašak, "Optimal parameterization of a PV and a battery system add-on for a consumer," in 2020 IEEE 11th International Symposium on Power Electronics for Distributed Generation Systems (PEDG), 2020, pp. 621626.

[13] J. Murphy, "Benders, nested benders and stochastic programming: An intuitive introduction," 2013. [Online]. Available:https://arxiv.org/abs/1312.3158v 